\title{
Blastic Plasmacytoid Dendritic Cell Neoplasm: A Case Report and Clinicopathological Review
}

\author{
Marwa Mohammed Abdel Fattah Zaki ${ }^{a}$, Khaled Zalata ${ }^{a}$, Amira Kamal El-Hawarya, c, \\ Noha Eisa ${ }^{\mathrm{b}}$, Shaimaa El Ashwah ${ }^{\mathrm{b}}$, Sameh Shamaa ${ }^{\mathrm{b}}$
}

\begin{abstract}
Blastic plasmacytoid dendritic cell neoplasm (BPDCN) is aggressive hematopoietic malignancy derived from the precursors of plasmacytoid dendritic cells. The present study reported a case of a 35-yearold BPDCN patient, who presented with scalp lesions without extracutaneous involvement of the lymph nodes (LNs), peripheral or bone marrow. Histopathological examination of scalp lesion revealed monomorphous diffuse infiltrate of small to medium-sized cells with irregular nuclear contours, pleomorphic nuclei, finely dispersed chromatin, inconspicuous nucleoli and scant amount of cytoplasm. Immunohistochemical staining showed diffuse positivity for CD45, CD4, CD 56, CD45 and negative for CD3, CD5, CD7, CD8, CD19, CD20, CD30, CD33, CD34, CD79a, CD99, CD117, TDT, and myeloperoxidase. Patient started treatment with acute lymphoblastic lymphoma protocol (Hyper-CVAD). Reevaluation after the second course showed marked regression of scalp lesion. The patient continued Hyper-CVAD protocol and planned for allogeneic stem cell transplant.
\end{abstract}

Keywords: Blastic; Plasmacytoid; Dendritic cell neoplasm

\section{Introduction}

Blastic plasmacytoid dendritic cell neoplasm (BPDCN) is an aggressive hematopoietic malignancy derived from the precursors of plasmacytoid dendritic cells, also known as professional type I interferon producing cells. This neoplasm is rare and has only recently been recognized as a distinct neoplastic entity [1].

This neoplasm was originally recognized in 1994 [2]. The uncertainty regarding its histogenesis and the challenges involved in classifying this hematopoietic neoplasm were re-

\footnotetext{
Manuscript submitted May 31, 2018, accepted July 24, 2018

aDepartment of Pathology, Faculty of Medicine, Mansoura University, Mansoura, Egypt

bedical Oncology Department, Oncology Center, Faculty of Medicine, Mansoura University, Mansoura, Egypt

${ }^{\mathrm{c} C}$ Corresponding Author: Amira Kamal El-Hawary, Department of Pathology, Faculty of Medicine, Mansoura University, Egypt.

Email: amira960@hotmail.com
}

doi: https://doi.org/10.14740/jh428w flected by the several changes of name, including blastic NKcell lymphoma, agranular CD4 natural killer cell leukemia, and agranular CD4/CD56 hematodermic neoplasm. Currently, the term blastic plasmacytoid dendritic cell neoplasm was introduced in 2008 by the updated WHO classification (fourth edition) [3]. The present study reported a case of a 35-year-old BPDCN patient, who presented with skin lesion without extracutaneous involvement of the lymph nodes (LNs), peripheral blood $(\mathrm{PB})$ or bone marrow $(\mathrm{BM})$.

\section{Case Report}

A 35 years old male patient presented to outpatient hematology clinic in Oncology Center, Mansoura University, with night fever, night sweating associated with history of scalp swelling started 3 months ago with progressive enlargement.

On clinical examination, the scalp lesion was a solitary bluish nodule measuring $3 \times 2 \mathrm{~cm}$ in size. The patient's biochemical profile showed that white blood cells count was 2.9 $\mathrm{k} / \mathrm{uL}$, hemoglobin level was $9.9 \mathrm{~g} / \mathrm{dL}$, and platelet count was $151 \mathrm{k} / \mathrm{uL}$. Liver and kidney functions tests were normal. Coagulation profile was normal. Viral markers for HCV, HBV, EBV and HIV were negative. LDH was $541 \mathrm{U} / \mathrm{L}$ which is high with moderate elevated ESR $(30 \mathrm{~mm} / \mathrm{h})$.

The patient underwent excisional biopsy of the scalp lesion. Histopathological examination revealed monomorphous non-epidermotropic diffuse infiltrate of small to medium-sized cells with irregular nuclear contours, pleomorphic nuclei, finely dispersed chromatin, inconspicuous nucleoli and scant amount of cytoplasm. This dense infiltrate was located in the dermis and hypodermis with perivascular and periadnexal aggregation, separated from the epidermis by a Grenz zone (Fig. $1,2)$.

Immunohistochemical (IHC) staining, performed on formalin fixed, paraffin-embedded tissue, showed strong diffuse positivity for CD45 (Fig. 3) CD4 (Fig. 4), CD56 (Fig. 5), CD68 (Fig. 6) and negative IHC staining for CD3, CD5, CD7, CD8, CD19, CD20, CD30, CD33, CD34, CD79 a, CD99, CD117, TDT, and myeloperoxidase. The proliferative index (Ki-67) was high; approximately $70 \%$.

BM aspirate showed a hyper cellular marrow with normal all series, and BM biopsy revealed reactive lymphoid follicles of mixed Band T lymphoid populations (as proved by IHC staining) with no infiltration by abnormal or neoplastic cells. CD34 and CD117 are within normal. CD56 was negative. 


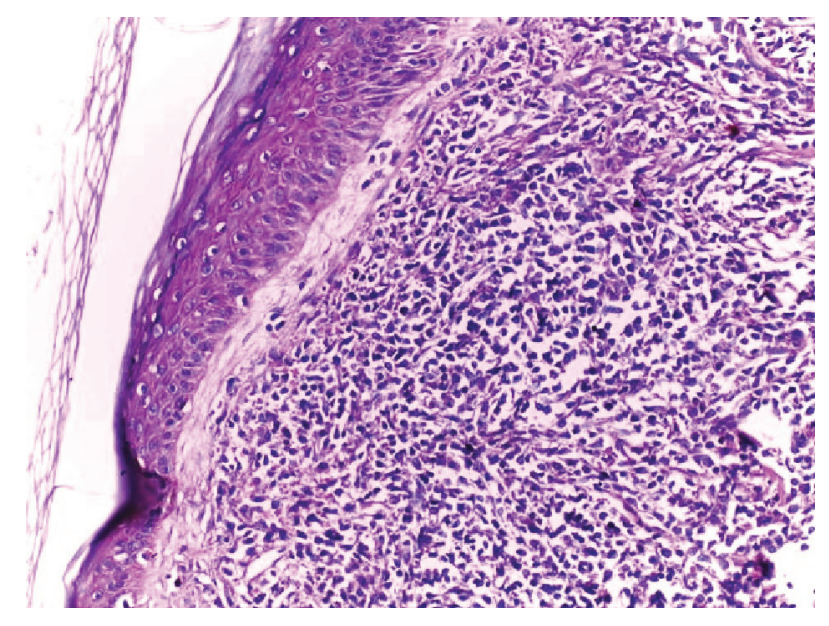

Figure 1. Infiltration of the dermis and hypodermis by monomorphous non-epidermotropic diffuse infiltrate of small to medium-sized cells with irregular nuclear contours. The cellular infiltrate is separated from the epidermis by a Grenz zone (H\&E, × 200).

The patient was diagnosed with BPDCN based on his clinical and histopathological findings. Patient started treatment with acute lymphoblastic lymphoma protocol (Hyper-CVAD). Reevaluation after the second course showed marked regression of scalp lesion with no detected other abnormal soft tissue masses and mildly enlarged reactive cervical lymph nodes. A repeat $\mathrm{BM}$ aspirate and biopsy revealed reduction of myeloid and erythroid series. CD20, CD3, CD4, CD34 and CD117 were within normal. CD56 was negative. The patient continued Hyper-CVAD protocol and planned for allogeneic stem cell transplant.

\section{Discussion}

The present study describes a middle-aged male of BPDCN presenting with skin lesions without extracutaneous involve-

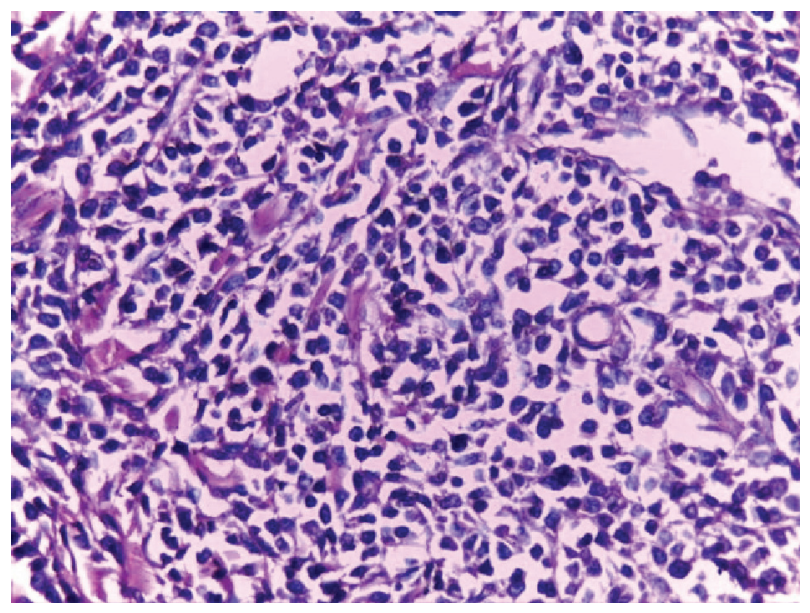

Figure 2. High power of the same case showed infiltrate of small to medium-sized cells with irregular nuclear contours, pleomorphic nuclei and scant amount of cytoplasm (H\&E, × 200).

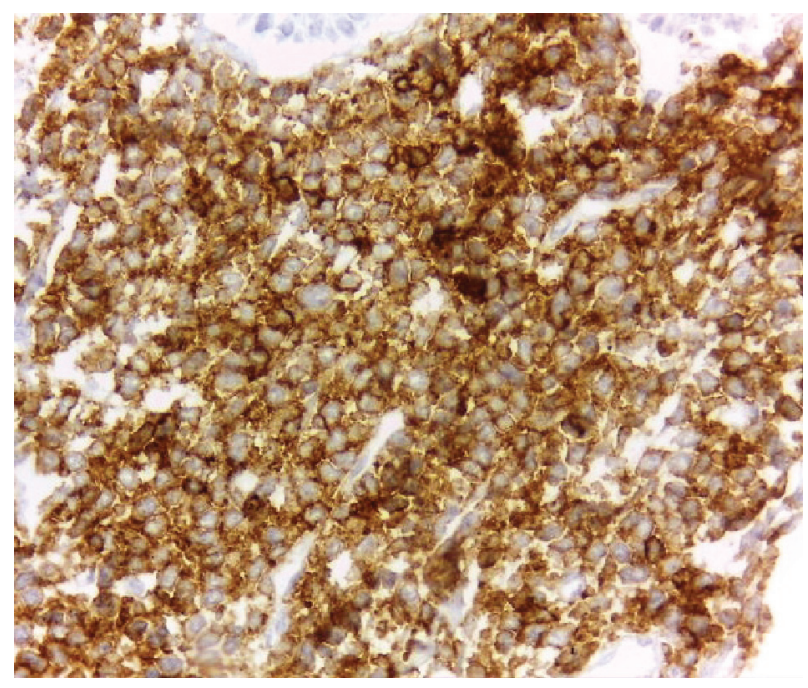

Figure 3. Strong membranous staining of the tumor cells for CD45 $(\mathrm{DAB}, \times 400)$.

ment of the LNs, PB or BM. The morphologic features of the neoplastic cells are typical for those of BPDCN. By IHC staining, the cells were CD45+, CD4+, CD56+ and lacked lineagespecific antigens. Unfortunately, markers specific for PDCs as CD123 IHC staining is not available in our country. So we depend on the characteristic morphologic features of the lesion with lack of epidermotropism and angio-destruction, negativity for lymphoid, NK and myeloid lineage-associated antigens, and Epstein-Barr virus negativity to establish a diagnosis of BPDCN and exclude other mimics. The unique aspect of the present case was that the patient was middle aged and the cutaneous lesion was not accompanied by extracutaneous involvements including LNs, PB and BM (stage I).

BPDCN typically occurs in elderly patients, with a mean age between 60 and 70 years; however, the disease may present at any age like our case who was 35 years. BPDCN is more prevalent in males, with a male to female ratio of 3:1 [4].

It has an aggressive clinical course with a poor prognosis.

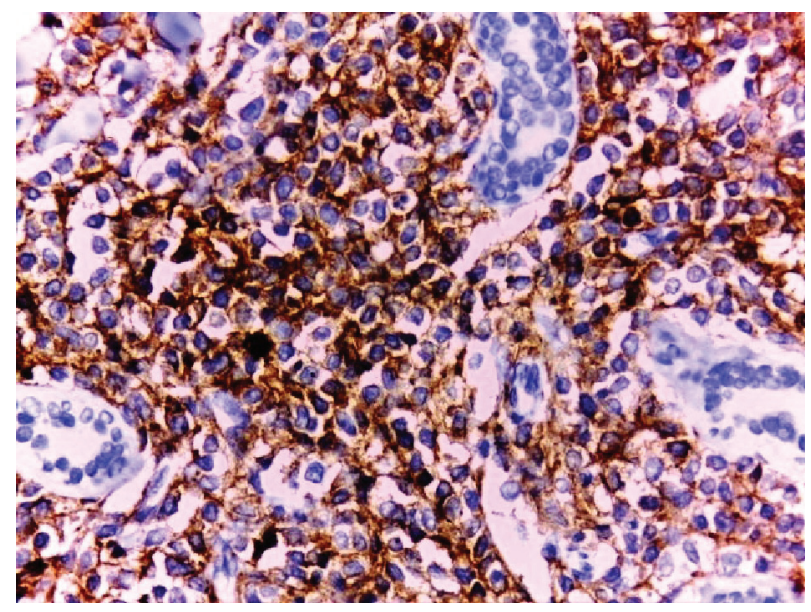

Figure 4. Diffuse positive membranous staining of the tumor cells for CD4 (DAB, $\times 400)$. Note periadnexal aggregation of the tumor cells. 


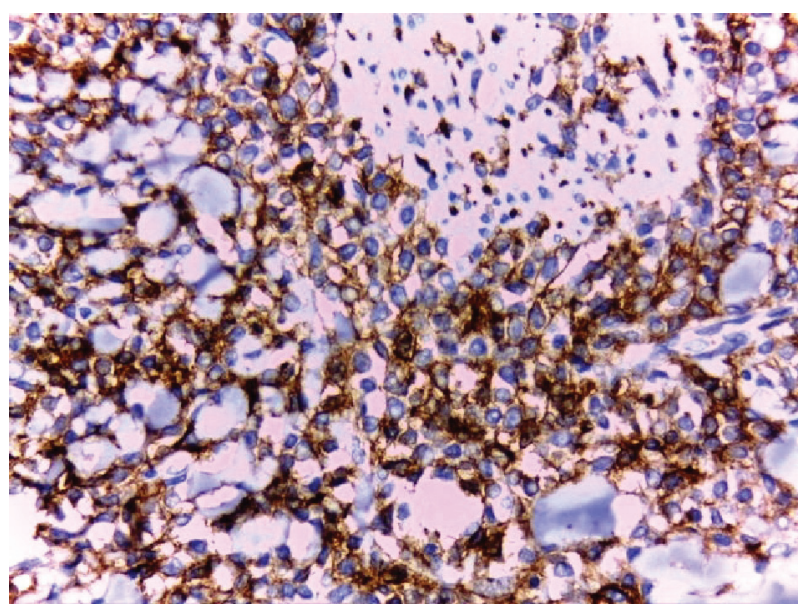

Figure 5. Strong membranous staining of the tumor cells for CD 56 $(\mathrm{DAB}, \times 400)$.

Ninety percent of cases presented with asymptomatic solitary/ multi-focal cutaneous reddish-brown nodules or bruise-like lesions. Extracutaneous disease includes regional lymphadenopathy, splenomegaly and hepatomegaly. Peripheral blood and BM involvement is variable and occurs in $60-90 \%$ of patients at diagnosis. Cytopenias are common, with thrombocytopenia being the most common, followed by anemia and neutropenia [5].

In the Ann Arbor staging system, most patients at diagnosis have stage IV disease, where as a few patients are classified as stage I; as in our case. It is extremely rare for BPDCN patients to present without cutaneous involvement, but even then, most of the patients will develop cutaneous lesions after an initial extracutaneous presentation $[5,6]$.

As the disease progresses, patients develop fulminant leukemia, particularly in the terminal stage of the disease, regardless of whether the patient presents with or without cutaneous lesions. In $10 \%$ to $20 \%$ of patients with BPDCN, coincident myelodysplasia is identified that can subsequently lead to the development of acute myelomonocytic leukemia or AML [4$6]$.

Histologically, BPDCN shows a diffuse, monomorphic infiltrate of medium-sized immature blastoid cells with round nuclei, finely dispersed chromatin, and absent or indistinct nucleoli. Typically, these cells involve the dermis and the subcutaneous adipose tissue but spare the epidermis. The typical monomorphous, blastic neoplastic cell morphology is evident in only $44.4 \%$ of cases. Histopathologic diagnosis can be challenging, contributing to the high rate of misdiagnosis of BPDCN [7].

Immunohistochemically, the diagnosis rests upon the demonstration of CD4 and CD56, together with markers more restricted to PDC (such as BDCA-2, CD123, TCL1, CD2AP and BCL11a) and negativity for lymphoid, NK and myeloid lineage-associated antigens [8]. Flow cytometry is preferred over IHC analysis as it allows for the examination of more markers and their intensity determination [9].

Terminal deoxynucleotidyl transferase (TdT) is expressed in approximately one-third of cases. Stem cell markers, includ-

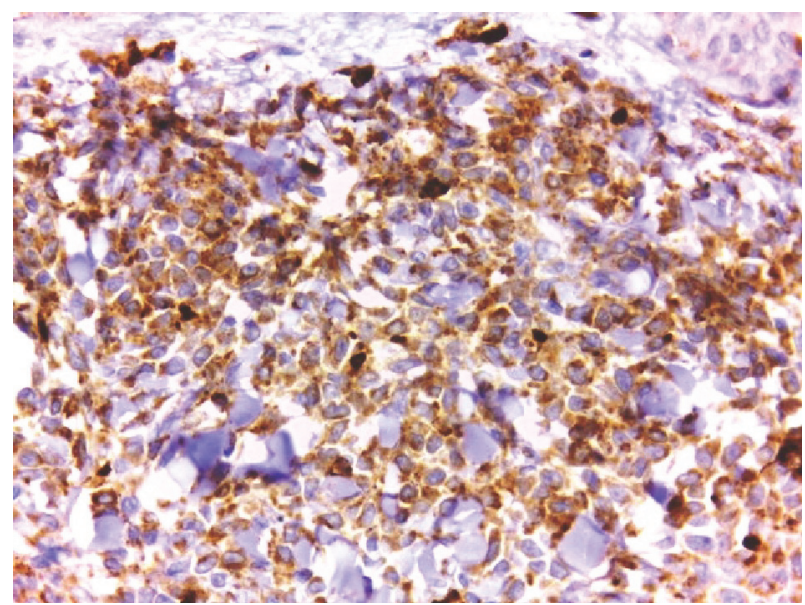

Figure 6. Positive cytoplasmic staining of the tumor cells for $C D 68$ (DAB, $\times 400)$.

ing CD34 and CD117, and Epstein-Barr virus-encoded small RNAs are negative [10]. CD7 and CD33 expression is common. T-cell markers (CD3, CD5) and B-cell markers (CD19, CD20, and CD79a) are not expressed. Typically, lysozyme and myeloperoxidase are negative. The Ki-67 labeling index of BPDCN reportedly ranges from $20 \%$ to $90 \%$. CD68 is noted in $50 \%$ of cases [11].

However, the neoplastic cells of BPDCN show a greater variability of morphologic and immunophenotypic features. Ascani et al and Aragrakos et al reported a CD4 negative variant of BPDCN $[12,13]$. Cases of CD56 negative and CD123 negative BPDCN also have been reported [7]. In such cases, BPDCN can be diagnosed based on morphology and complete immunophenotypic profile [11].

Patients with cutaneous T-cell lymphoma frequently present with skin lesions and blood involvement, but the disease can be differentiated from BPDCN based on morphology, disproportionate epidermotropism, and mature T-cell immunophenotype with a lack of CD56 expression. Extranodal NK/ T-cell lymphoma can manifest with skin lesions and the expression of the $\mathrm{CD} 4+/ \mathrm{CD} 56+$ immunophenotype. This rare aggressive malignancy can be differentiated from BPDCN by demonstrating Epstein-Barr virus positivity via in situ hybridization using Epstein-Barr virus-encoded small ribonucleic acids [11].

Researchers debate whether tumor cells are originally sited in the BM or skin. Unresolved questions remain regarding its histogenesis and aggressive clinical presentation that commonly affect both sites either consecutively or simultaneously. Furthermore the existence of rapidly disseminating disease cases and those with primarily cutaneous disease and indolent progression raise questions to whether this represents two stages of a disease spectrum or two different CD4+CD56+ malignancies [14].

Currently, there is no standardized treatment for this neoplasm; and therapy varies based on institutional preference. Initially, cutaneous lesions can be treated with focal radiation, oral glucocorticoids, or chemotherapy. Although skin-limited disease has been reported to have a better prognosis than ex- 
tracutaneous disease, it should be treated aggressively since leukemic dissemination is inevitable [11-15].

Patients with BPDCN are usually treated with regimens used for other hematopoietic malignancies such as nonHodgkin lymphoma (CHOP) and acute myeloid leukemia and lymphoma (cyclophosphamide/vincristine/doxorubicin/ dexamethasone alternating with methotrexate and cytarabine (hyper-CVAD)). Initially, patients commonly respond well to chemotherapy with complete resolution of skin lesions, but frequently relapse as chemotherapeutic drug resistance develops $[16,17]$.

Due to the aggressive nature of this malignancy, allogeneic bone marrow transplantation (BMT) is one of the best therapeutic strategies to effectively treat BPDCN. However, advanced age and comorbidities in many patients with BPDCN limit the use of allogeneic BMT. Studies demonstrate that the best long-term prognosis is in younger patients who undergo allogeneic BMT after chemotherapy induced remission [15-17].

\section{Funding}

This work had no specific funding.

\section{Disclosures}

There were no financial disclosures from any author.

\section{References}

1. Herling M, Jones D. CD4+/CD56+ hematodermic tumor: the features of an evolving entity and its relationship to dendritic cells. Am J Clin Pathol. 2007;127(5):687-700.

2. Adachi M, Maeda K, Takekawa M, Hinoda Y, Imai K, Sugiyama S, Yachi A. High expression of CD56 (NCAM) in a patient with cutaneous CD4-positive lymphoma. Am J Hematol. 1994;47(4):278-282.

3. Facchetti F, Jones DM, Petrella T. Blastic plasmacytoid dendritic cells neoplasm. In: Swerdlow SH, Campo E, Harris NL, Jaffe ES, Pileri SA, Stein H, Thiele J, ed. WHO classification of Tumours of Haematopoietic and Lymphoid Tissues (ed 4th). Lyon, France: International Agency for Research on Cancer 2008:145-147.

4. Magro CM, Porcu P, Schaefer J, Erter JW, Furman RR, Shitabata PK, Crowson AN. Cutaneous CD4+ CD56+ hematologic malignancies. J Am Acad Dermatol. 2010;63(2):292-308.

5. Petrella T, Comeau MR, Maynadie M, Couillault G, De Muret A, Maliszewski CR, Dalac S, et al. 'Agranular CD4+ CD56+ hematodermic neoplasm' (blastic NK- cell lymphoma) originates from a population of CD56+ precursor cells related to plasmacytoid monocytes. Am J Surg Pathol. 2002;26(7):852-862.

6. Zhang YW, Zhong JH, Chen XL, Xiao F, Chen FY. Blastic plasmacytoid dendritic cell neoplasm: A case report and literature review. Exp Ther Med. 2016;12(1):319322.

7. Cota C, Vale E, Viana I, Requena L, Ferrara G, Anemona L, Metze D, et al. Cutaneous manifestations of blastic plasmacytoid dendritic cell neoplasm-morphologic and phenotypic variability in a series of 33 patients. Am J Surg Pathol. 2010;34(1):75-87.

8. Facchetti F, Ungari M, Marocolo D, Lonardi S, Vermi W. Blastic plasmacytoid dendritic cell neoplasm. Hematol Meeting Rep. 2009;3(3):1-3.

9. Tsagarakis NJ, Kentrou NA, Papadimitriou KA, Pagoni M, Kokkini G, Papadaki H, Pappa V, et al. Acute lymphoplasmacytoid dendritic cell (DC2) leukemia: results from the Hellenic Dendritic Cell Leukemia Study Group. Leuk Res. 2010;34(4):438-446.

10. Li HS, Watowich SS. Diversification of dendritic cell subsets: Emerging roles for STAT proteins. JAKSTAT. 2013;2(4):e25112.

11. Riaz W, Zhang L, Horna P, Sokol L. Blastic plasmacytoid dendritic cell neoplasm: update on molecular biology, diagnosis, and therapy. Cancer Control. 2014;21(4):279289.

12. Ascani S, Massone C, Ferrara G, Rongioletti F, Papini M, Pileri S, Cerroni L. CD4-negative variant of CD4+/ CD56+ hematodermic neoplasm: description of three cases. J Cutan Pathol. 2008;35(10):911-915.

13. Argyrakos T, Rontogianni D, Karmiris T, Kapsimali V, Grigoriou E, Tsantekidou M, Naum C, et al. Blastic natural killer (NK)-cell lymphoma: report of an unusual CD4 negative case and review of the CD4 negative neoplasms with blastic features in the literature. Leuk Lymphoma. 2004;45(10):2127-2133.

14. Lencastre A, Cabete J, Joao A, Farinha P, Ferreira G, Lestre S. Blastic plasmacytoid dendritic cell neoplasm. An Bras Dermatol. 2013;88(6 Suppl 1):158-161.

15. Gera S, Dekmezian MS, Duvic M, Tschen JA, Vega F, Cho-Vega JH. Blastic plasmacytoid dendritic cell neoplasm: evolving insights in an aggressive hematopoietic malignancy with a predilection of skin involvement. Am J Dermatopathol. 2014;36(3):244-251.

16. Xin X, Tian M, Huang L, Wang G, Cheng L, Liu Z, Dong $\mathrm{P}$, et al. Cutaneous lesions from a blastic plasmacytoid dendritic cell neoplasm: a case report and literature review. Int J Clin Exp Med. 2016;9:5017-5022.

17. Grushchak S, Joy C, Gray A, Opel D, Speiser J, Reserva $\mathrm{J}$, Tung R, et al. Novel treatment of blastic plasmacytoid dendritic cell neoplasm: A case report. Medicine (Baltimore). 2017;96(51):e9452. 